\title{
Contradiction Analysis for Constraint-based Random Simulation*
}

\author{
Daniel Große ${ }^{1} \quad$ Robert Wille $^{1} \quad$ Robert Siegmund $^{2} \quad$ Rolf Drechsler $^{1}$ \\ ${ }^{1}$ Institute of Computer Science, University of Bremen, 28359 Bremen, Germany \\ \{grosse,rwille,drechsle\}@informatik.uni-bremen.de \\ ${ }^{2}$ AMD Saxony LLC \& Co. KG, Dresden Design Center, 01330 Dresden, Germany \\ robert.siegmund@amd.com
}

\begin{abstract}
Constraint-based random simulation is state-of-the-art in verification of multi-million gate industrial designs. This method is based on stimulus generation by constraint solving. The resulting stimuli will particularly cover corner case test scenarios which are usually hard to identify manually by the verification engineer. Consequently, constraintbased random simulation will catch corner case bugs that would remain undetected otherwise. Therefore, the quality of design verification is increased significantly. However, in the process of constraint specification for a specific test scenario, the verification engineer is faced with the problem of over-constraining, i.e. the overall constraint specified for a test scenario has no solution. In this case the root cause of the contradiction has to be identified and resolved. Given the complexity of constraints used to describe test scenarios, this can be a very time-consuming process.

In this paper we propose a fully automated contradiction analysis method. Our method determines all "non relevant" constraints and computes all reasons that lead to the over-constraining. Thus, we pinpoint the verification engineer to exactly the sets of constraints that have to be considered to resolve the over-constraining. Experiments have been conducted in a real-life SystemC-based verification environment at AMD Dresden Design Center. They demonstrate a significant reduction of the constraint contradiction debug time.
\end{abstract}

\section{Introduction}

The continued advance of circuit fabrication technology that persisted over the last 30 years now allows the integration of more than 1 billion transistors in System-on-Chip (SoC) designs. The development of SoCs of such complexity leads to enormous challenges in Computer-Aided Design (CAD), especially in the area of design verification, which needs to ensure the functional correctness of a design. Because the capacity of formal verification is limited, simulation is still the most frequently used verification technique [22].

In directed simulation explicitly specified stimulus patterns (e.g. written by verification engineers) are applied to the design. Each of those patterns stimulates a very specific design functionality (called a verification scenario) and the response of the design is compared thereafter with the expected result. Due to project time constraints, it is inherent for directed simulation that only a limited number of such scenarios will be verified.

With random simulation these limitations are compensated. Random stimuli are generated as inputs for the design. For example, to verify the communication over a bus, random addresses, and random data are computed.

* This research work was supported in part by the German Federal Ministry of Education and Research (BMBF) in the project URANOS under the contract number $01 \mathrm{M} 3075$.
A substantial time reduction for the creation of simulation scenarios is achieved by constraint-based random simulation (see e.g. [2, 22]). Here, the stimuli are generated directly from specified constraints by means of a constraint solver, i.e. stimulus patterns are selected by the solver which satisfy the constraints. The resulting stimuli will also cover test scenarios for corner cases that may be difficult to generate manually. As a consequence, design bugs will be found that might otherwise remain undetected, and the quality of design verification increases substantially.

For constraint-based random simulation several approaches have been proposed (see e.g. [23, 4, 11, 21, 12]). However, a major problem that arises when stimuli are specified in form of constraints is over-constraining, i.e. the constraint solver is not able to find a valid solution for the given set of constraints. Whenever such a contradiction occurs in a constraint-based random simulation run, this run has to be terminated as no valid stimulus patterns can be applied. Note that over-constraining may not necessarily happen at the very beginning of the simulation run, as modern testbench languages such as SystemVerilog [9] allow the addition of constraints dynamically during simulation. In any case of over-constraining the verification engineer has to identify the root cause of the constraint contradiction. As this is usually done manually by either code inspection or trial-and-error debug, it is a tedious and time-consuming process.

To the best of our knowledge in this work we propose the first non-trivial algorithm for contradiction analysis in constraint-based random simulation. In the area of constraint satisfaction problems methods for diagnosing over-constrained problems have been introduced (see e.g. $[1,16])$. These methods aim to find a solution for the over-constrained problem by relaxing constraints according to a given weight for each constraint. In the considered problem no weights are available. Also, the approaches do not determine all minimal reasons that cause the overall contradiction. In contrast, Yuan et al. proposed an approach to locate the source of a conflict using a kind of exhaustive enumeration [22]. But since a very large runtime of this method is supposed - neither an implementation nor experiments are provided - they recommend to build an approximation. In the domain of Boolean Satisfiability (SAT) a somewhat similar problem can be found: computing an unsat core of an unsatisfiable formula, i.e. to identify an unsatisfiable sub-formula of the overall formula $[5,24]$. However, to obtain a minimal reason the much more complex problem of a minimal unsat core has to be considered $[15,7,14]$. Furthermore, all minimal unsat cores are required to determine all contradictions. In general this is very time consuming (see e.g. [13]).

In this paper we propose a fully automatic technique for analyzing contradictions in constraint-based random simulation. The basic idea is as follows: The overall constraint is reformulated such that (contradicting) constraints can be disabled by introducing new free variables. Next, an ab- 


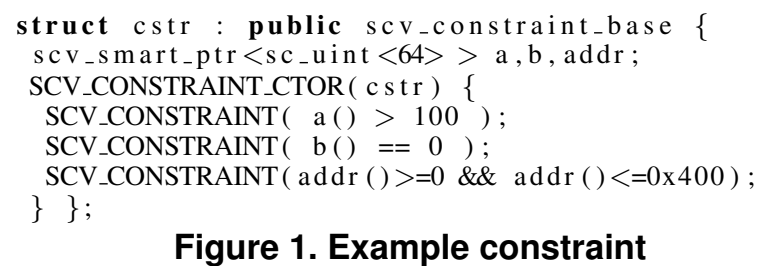

straction is computed that forms the basis for the following steps. First, the self-contradicting constraints are identified. Then, all "non relevant" constraints are determined. Finally, for the remaining constraints - typically only a very small set - a detailed analysis is performed. In total our approach identifies all reasons of the over-constraining, i.e. all minimal constraint combinations that lead to a contradiction of the overall constraint. As shown by experiments in a verification environment of AMD Dresden Design Center (DDC), the debugging time is reduced significantly. The verification engineer completely understands what causes the over-constraining and can resolve the contradictions in one single step.

\section{SystemC Verification Library}

This section briefly reviews the SystemC Verification (SCV) library that is used for constraint-based random simulation in this work. The SCV library was introduced in 2002 as an open source C++ class library [20, 17, 10] on top of SystemC $[19,8]$. In the following we focus only on the basic features of the SCV library for constraint-based random simulation.

Using the SCV library, constraints are modeled in terms of $\mathrm{C}++$ classes. That way constraints can be hierarchically layered using $\mathrm{C}++$ class inheritance. In detail a constraint is derived from the scv_constraint_base class. The data to be randomized is specified as SCV_smart_ptr variables.

An example of an SCV constraint is shown in Figure 1. The name of the constraint is cstr. Here, the three 64 bit unsigned integer variables $a, b$, and $a d d r$ are randomized. The conditions on the variables $a, b$, and $a d d r$ are defined by expressions in the respective $S C V_{-} C O N S T R A I N T$ () macro.

Internally, a constraint in the SCV library is represented by the corresponding characteristic function, i.e. the function is true for all solutions of the constraint. This characteristic function of a constraint is represented as a Binary Decision Diagram (BDD), a canonical and compact data structure for Boolean functions [3]. For stimuli generation a weighting algorithm is applied for the constraint BDD to guarantee a uniform distribution of all constraint solutions and hence maximizing the chance for entering unexplored regions of the design state space. As BDD package CUDD [18] is used in the SCV library.

\section{Contradiction Analysis}

In this section first the considered problem, that is the contradiction of constraints, is formalized. Then, we present concepts for the contradiction analysis approach.

\subsection{Problem Formulation}

Before the problem is formulated we define the type of constraints that are considered in this paper.

Definition 1. A constraint is a Boolean function over variables from the set of variables $V$. For the specification of a constraint, the typical HDL operators such as e.g. logic $A N D$, logic OR, arithmetic operators, and relational operators can be used.
Usually a constraint consists of a conjunction of other constraints. We formalize the resulting overall constraint in the following definition.

Definition 2. An overall constraint is defined as

$$
C=\bigwedge_{i=0}^{n-1} C_{i}
$$

where $C_{i}$ are constraints according to Definition 1.

In practice, the conjunction is built by the explicit use of several SCV_CONSTRAINT() macros or by applying inheritance, i.e. parts of the constraints are defined in a base class and inherited in the actual constraint. Note that this is not specific to constraint-based random simulation using the SCV library. In fact, the same principles are found, for example, in the random constraints of System Verilog [9].

During the specification of complex non-trivial constraints, the problem of over-constraining arises:

Definition 3. An overall constraint $C$ is over-constrained or contradictory iff $C$ is not satisfiable, i.e. $C$ evaluates to 0 for all assignments to the constraint variables.

Typically, if $C$ is over-constrained the verification engineer has to manually identify the reason for the overconstraining. This process can be very time-consuming because several cases are possible. For example, one of the constraints $C_{i}$ may have no solution. Another reason for a contradiction may be that the conjunction of some of the constraints $C_{i}$ leads to 0 . In the following the term reason as used in the rest of this paper is defined.

Definition 4. A reason for a contradictory overall constraint $C$ is the set $R=\left\{C_{i_{1}}, C_{i_{2}}, \ldots, C_{i_{k}}\right\} \subseteq$ $\left\{C_{0}, C_{1}, \ldots, C_{n-1}\right\}$ with the two properties:

1. The constraints in $R$ form a contradiction, i.e. the conjunction $C_{i_{1}} \wedge C_{i_{2}} \wedge \ldots \wedge C_{i_{k}}$ always evaluates to 0 . Therefore the overall constraint $C$ is contradictory.

2. Removing an arbitrary constraint from $R$ resolves the contradiction, i.e. minimality of $R$ is required.

Often the root of over-constraining results from more than one contradiction, i.e. there is more than one reason. If in this case only one reason is identified by the verification engineer, the constraint solver has to solve the fixed constraint again, but still there is no solution.

Based on these observations, the following problem is considered in this paper:

How can we efficiently compute all minimal reasons for an over-constraining and thereby support the verification engineer in constraint debugging?

Analyzing the contradictions in the overall constraint $C$ and presenting all reasons is facilitated by our approach. In particular excluding all constraints which are not part of a contradiction reduces the debugging time significantly.

\subsection{Concepts for Contradiction Analysis}

The general idea of the contradiction analysis approach is as follows: The overall constraint $C$ is reformulated such that the conflicting constraints can be disabled by the constraint solver and $C$ becomes satisfiable. By analyzing the logical dependencies of the disabled constraints, we can identify all reasons for the over-constraining. 


$$
\begin{aligned}
C_{0} & \Leftrightarrow \mathrm{b}()<3 \& \& \mathrm{~b}()==7 \\
C_{1} & \Leftrightarrow \mathrm{a}()+\mathrm{b}()==\mathrm{c}() \\
C_{2} & \Leftrightarrow \mathrm{a}()<6 \\
C_{3} & \Leftrightarrow \mathrm{a}()==5 \\
C_{4} & \Leftrightarrow \mathrm{a}()==10 \\
C_{5} & \Leftrightarrow \mathrm{d}()==8 \\
C_{6} & \Leftrightarrow \mathrm{d}()>10
\end{aligned}
$$

(a)

\section{Figure 2. Contradictory constraint}

Definition 5. Let $C$ be over-constrained. Then the reformulated constraint $C^{\prime}$ is built by introducing a new free variable $s_{i}$ for each constraint $C_{i}$ and substituting each constraint $C_{i}$ with an implication from $s_{i}$ to $C_{i}$. That is,

$$
C^{\prime}=\bigwedge_{i=0}^{n-1}\left(s_{i} \rightarrow C_{i}\right) .
$$

For the reformulated constraint $C^{\prime}$ the following holds:

1. If $s_{i}$ is set to 1 , then the constraint $C_{i}$ is enabled.

2. If $s_{i}$ is set to 0 , then the constraint $C_{i}$ is disabled because $C_{i}$ can evaluate to 0 or 1 .

Note that the usage of an implication is crucial. If an equivalence is used instead of an implication, $s_{i}=0$ would imply the negation of $C_{i}$.

Example 1. Figure 2(a) shows a constraint $C$ which is over-constrained. Reformulating $C$ to $C^{\prime}$ avoids the overconstraining because a constraint $C_{i}$ may be disabled by assigning $s_{i}$ to 0 . The table in Figure 2(b) gives all assignments to $s_{i}$ such that the reformulated overall constraint $C^{\prime}$ evaluates to $1 .^{1}$ That is, the table shows which constraints have to be disabled to get a valid solution. For example, from the first row it can be seen that disabling $C_{0}, C_{2}, C_{3}$, and $C_{5}$ avoids the contradiction.

Based on the reformulation the verification engineer is able to avoid the over-constraining. But to understand what causes the over-constraining, i.e. to identify the reason of each contradiction, a more detailed analysis is required. Here, two properties of the assignment table obtained from the reformulated overall constraint can be exploited.

Note that for simplicity we always refer to the assignment table in the presentation. As shown later in the implementation the assignment table needs not to be build explicitly.

Property 1. The value of variable $s_{i}$ is 0 for all solutions (i.e. in each row of the table) iff the respective constraint $C_{i}$ is self-contradictory (that is $C_{i}$ has no solution).

Proof. $\Rightarrow$ : We show this by contraposition: If $C_{i}$ has at least one solution, then there is a row where $s_{i}$ is 1. Obviously this solution (row) can be constructed by assigning 1 to $s_{i}$ and 0 to $s_{j}$ for $j \neq i$, because $\left(s_{i} \rightarrow C_{i}\right)=\bar{s}_{i} \vee C_{i}=0 \vee C_{i}=C_{i}=1$ and $\left(s_{j} \rightarrow C_{j}\right)=\bar{s}_{j} \vee C_{j}=1 \vee C_{j}=1$ for $j \neq i$.

$\Leftarrow$ : To satisfy $C^{\prime}$ each element of the conjunction must evaluate to 1 , so $\left(s_{i} \rightarrow C_{i}\right)=\bar{s}_{i} \vee C_{i}$. Since $C_{i}$ has no solution $\left(C_{i}\right.$ is always 0$) s_{i}$ must be 0 .

Thus, each constraint $C_{i}$ whose $s_{i}$ variable is always assigned to 0 , is a reason for the contradictory overall constraint $C$.

\footnotetext{
${ }^{1}$ Here '-' denotes a don't care, i.e. the value of $s_{i}$ can be either 0 or 1 . The table is derived from a symbolic BDD representation of all solutions for the $s_{i}$ variables after abstraction of all other variables.
}

Property 2. The value of variable $s_{i}$ is don't care for all solutions (i.e. for all rows of the table) iff the constraint $C_{i}$ is never part of a contradiction of $C$.

Proof. $\Rightarrow$ : This property is shown by contradiction. Assume that $s_{i}$ is don't care for all solutions and $C_{i}$ is part of a contradiction. Then, without loss of generality there has to be another satisfiable constraint $C_{j}$ such that $C_{i} \wedge C_{j}=0$. $^{2}$ If $s_{j}$ is set to 1 and all other constraints $C_{k}$ with $k \neq j$ are disabled by $s_{k}=0$, then $C^{\prime}$ is 1 . However, switching $s_{i}$ to 1 is not possible due to the conflict of $C_{i}$ and $C_{j}$. But this contradicts the assumption that the value of $s_{i}$ is don't care for all solutions.

$\Leftarrow$ : Because the constraint $C_{i}$ is never part of a contradiction, $C_{i}$ can be enabled or can be disabled. In other words, $s_{i}$ can be set to 0 and also to 1 for each solution of the overall constraint, which is equivalent to $s_{i}$ is don't care.

Thus, each constraint $C_{i}$ whose $s_{i}$ variable is always don't care, is not part of a reason for the contradictory overall constraint. Therefore these constraints are not presented to the verification engineer and can be left out in the next steps.

Example 2. Consider again Example 1. Because the value of $s_{0}$ is 0 for all solutions, $C_{0}$ is self-contradictory. Thus, $R_{0}=\left\{C_{0}\right\}$ is a reason for $C$. Since the value of $s_{1}$ is always don't care, $C_{1}$ is never part of a contradiction. As a result the first two constraints can be ignored in the further analysis.

Note that the overall constraint of the example in Figure 2(a) has been specified to demonstrate the two properties. In practice, the number of constraints that are never part of a contradiction is considerably larger. Thus, applying Property 2 reduces the debugging effort significantly because each "non relevant" constraint does not have to considered anymore by the verification engineer.

In fact, all remaining constraints (if there are any) are part of at least one contradiction. Furthermore, since selfcontradictory constraints have been filtered out by Property 1 only a conjunction of two or more constraints causes a contradiction. Now the question is, how can we identify the minimal contradicting conjunctions of the remaining constraints, i.e. the reasons?

Example 3. Again Example 1 is considered. The constraints $C_{0}$ and $C_{1}$ have been handled already according to Property 1 and Property 2. Now, the conjunction of two or more of the remaining constraints, $C_{2}, C_{3}, C_{4}, C_{5}$, and $C_{6}$, causes a contradiction. Only identifying the product of all these constraints certainly does not help to resolve the conflict easily. In contrast, the over-constraining can only be fixed if the different contradictions are understood. But this requires the computation of all minimal reasons according to Definition 4. In the example, three reasons can be found in total: $R_{1}=\left\{C_{2}, C_{4}\right\}$ and $R_{2}=\left\{C_{3}, C_{4}\right\}$ which overlap as well as $R_{3}=\left\{C_{5}, C_{6}\right\}$ which is independent of the two before.

To find the minimal reason for each contradiction, all constraint combinations are tested for a contradiction starting with the smallest conjunction. For each tested combination the respective $s_{i}$ variables are set to 1 . Thus, if the conjunction $C_{i_{1}} \wedge \ldots \wedge C_{i_{k}}$ leads to a contradiction

\footnotetext{
${ }^{2}$ According to Property 1 both constraints $C_{i}$ and $C_{j}$ have at least one solution.
} 
(1) ContradictionAnalysis(BDD $C^{\prime}$, set $V$ )

(2) // abstraction

(3) $C^{\prime \prime}=\exists v_{1}, \ldots, \exists v_{|V|} C^{\prime}$

(4) // initialization

(5) $\mathcal{R}=\emptyset ; / /$ reasons of contradictions

(6) $S=\emptyset ; / / s_{i}$ variables for detailed anaysis

(7) // test properties

(8) for $(i=0 ; i<n ; i++)$

if $\left(\left(C^{\prime \prime} \wedge s_{i}=1\right) \equiv 0\right)$ $/ / C_{i}$ is self-contradictory $\mathcal{R}=\mathcal{R} \cup\left\{\left\{s_{i}\right\}\right\}$; else

// $C_{i}$ is selected for detailed analysis $S=S \cup\left\{s_{i}\right\}$;

// detailed analysis

for each $(X \in \mathcal{P}(S))$

// from the smallest to the largest

if $\left(\exists X^{\prime} \in \mathcal{R}: X^{\prime} \subset X\right)$

// ensure minimality

continue;

if $\left.\left(\left(C^{\prime \prime} \wedge \bigwedge_{s_{i} \in X} s_{i}=1\right) \equiv 0\right)\right)$

// subset over-constrains $C$

$\mathcal{R}=\mathcal{R} \cup\{X\}$

return $\mathcal{R}$; else if $\left(\left(C^{\prime \prime} \wedge s_{i}=0\right) \equiv\left(C^{\prime \prime} \wedge s_{i}=1\right)\right)$ $/ / C_{i}$ is not responsible for over-constr.

Figure 3. Overall algorithm

$\left(\left(s_{i_{1}}=1\right) \wedge \ldots \wedge\left(s_{i_{k}}=1\right) \wedge C^{\prime} \equiv 0\right)$, then this combination is a reason for $C$. The minimality is ensured by building the constraint combinations in ascending order with respect to their size and skipping each superset of a previously found reason. Since the overall problem has already been simplified by exploiting Property 1 and Property 2 , the combination based procedure has to be applied only for a small set of constraints, i.e. the remaining ones. This is the key to the efficiency of the overall contradiction analysis procedure.

The next section presents the details on the implementation of the overall contradiction analysis approach.

\section{Implementation}

As already mentioned earlier, the SCV library uses BDDs for the representation of constraints. More precisely the characteristic function of the overall constraint is represented as a BDD. This characteristic function is true for all solutions of the constraint, false otherwise. We implemented the contradiction analysis approach using the SCV library. Therefore our implementation is "BDD driven".

The pseudo-code of the contradiction analysis approach is shown in Figure 3. As input the approach starts with the BDD representation of the reformulated constraint $C^{\prime}$ and the set of all constraint variables $V$. At first, all constraint variables are existentially quantified from the reformulated constraint (line 3). Thus, the resulting function $C^{\prime \prime}$ only depends on the $s_{i}$ variables. In other words, this function is the symbolic representation of the assignment table described in the previous section. In general the quantified $\mathrm{BDD}$ is much more compact than the BDD for the reformulated constraint. Thus, the following BDD operations can be executed very fast.

After quantification the two sets $\mathcal{R}$ and $S$ are initialized to the empty set. $\mathcal{R}$ stores all reasons that are found. Note that for simplicity $\mathcal{R}$ contains the sets of the corresponding $s_{i}$ variables of a reason, not the constraints itself. The set $S$ is used to save all $s_{i}$ variables that are passed to the detailed analysis later. So this set corresponds to the remaining constraints. Then, for each constraint $C_{i}$ it is checked if $C_{i}$ is either self-contradictory (line 9 ) or never part of a contradiction (line 12) according to Property 1 and Property 2. In the former case the respective $s_{i}$ variable is added to the set of reasons $\mathcal{R}$ (line 11). Both checks are conducted on the quantified representation $C^{\prime \prime}$ of the reformulated constraint, that is:

- To check if $s_{i}$ is 0 for all solutions (see Property 1) the conjunction $C^{\prime \prime} \wedge s_{i}=1$ is carried out. If the result is the constant zero-function, $s_{i}$ is never 1 in any solution, i.e. $s_{i}$ is always zero. Thus, $C_{i}$ becomes a reason.

- The check if $s_{i}$ is don't care in all solutions (see Property 2$)$ is carried out by $\left(C^{\prime \prime} \wedge s_{i}=0\right) \equiv\left(C^{\prime \prime} \wedge s_{i}=1\right)$. If the respective BDDs are equal, it has been shown that $s_{i}$ is don't care, since regardless of the value of $s_{i}$ the solutions are identical. Therefore, the constraint $C_{i}$ is not relevant for a contradiction and thus neither added to the set $\mathcal{R}$ nor to the set $\mathcal{S}$.

If both properties cannot be applied (line 14), then the respective constraint $C_{i}$ is part of a contradiction caused by the conjunction of $C_{i}$ with one or more other constraints Thus, $C_{i}$ is passed to the detailed analysis by inserting the respective $s_{i}$ into $S$ (line 16).

Finally, the detailed analysis for all elements in $S$ - the remaining constraints - is performed (line 18 to 25). First, the power set $\mathcal{P}(S)$ of $S$ is created resulting in all subsets (i.e. combinations) of constraints considered for detailed analysis. Note that we exclude the empty set as well as all sets which only contain one element (this is already covered by Property 1) from the power set. Furthermore, during the construction the elements of the power set are ordered according to their cardinality. Then, for each subset $X$ (i.e. for each combination) the conjunction of the respective constraints is tested for a contradiction. Therefore, the conjunction of the current combination $X$-represented as a cube of all variables $s_{i} \in X-$ and $C^{\prime \prime}$ is created, i.e. all respective constraints $C_{i}$ are enabled (line 23). If the conjunction leads to a contradiction, then $X$ is a reason and thus, $X$ is added to $\mathcal{R}$ (line 25 ). To ensure minimality each contradiction test of a subset $X$ is only carried out if no reason $X^{\prime} \in R$ exists such that $X^{\prime} \subset X$ (line 20-22), i.e. no subset of $X$ has already been identified as reason for a contradiction (see also Definition 4).

In summary, the presented contradiction analysis procedure computes all minimal reasons $\mathcal{R}$ of a contradictory overall constraint $C$. First, the proposed reformulation of the overall constraint allows a representation where all contradictory constraints can be disabled. From this representation a much more compact one is computed by quantification. All following operations have to be carried out on this representation only. Then, the two properties are applied which significantly reduces the problem size since only $2^{n-|Z|-|D C|}$ instead of all $2^{n}$ subsets have to be considered in the detailed analysis ( $Z$ denotes the set of selfcontradictory constraints, and $D C$ denotes the set of constraints, which are not part of a contradiction). In practice, especially the number of "non relevant" constraints that belong to the set $D C$ is very large, so the input for the detailed analysis shrinks considerably.

\section{Experimental Evaluation}

This section provides experimental results for the contradiction analysis. We show the efficiency of our approach by 
several testcases. Finally, the application of our approach in an industrial setting is presented.

In all examples the partitioning of the constraints is given according to the specification in the constraint classes, i.e. each $C_{i}$ in the following corresponds to a separate SCV_CONSTRAINT() macro (see also Section 3.1). The contradiction analysis is started by an additional commandline switch and runs fully automatic in the SCV library environment.

\subsection{Effect of Property 1 and Property 2}

Applying the two properties introduced in Section 3.2 significantly reduces the complexity of the contradiction analysis since each matched constraint can be excluded from further considerations. To show the increasing efficiency we tested our approach for several examples which contain some typical overconstraining errors (e.g. typos, contradicting implications, hierarchical contradictions, etc.).

For the considered constraints we give some statistics in Table 1. In the first column a number to identify the testcase is given. Then, in the next columns information on the constraint variables and their respective sizes are provided. Finally, the total number of constraints is given. The results after application of our contradiction analysis are shown in Table 2. The first four columns give some information about the testcase, i.e. the number of constraints in total $(n)$, the number of contradictions/reasons $(|\mathcal{R}|)$, and the runtime in CPU seconds needed to construct the BDD in the SCV library (BDD TIME). The next columns provide the results for the trivial analysis approach without (W/O PROPERTIES) and with the application of the properties (WITH PROPERTIES), respectively. Here the number of checks in the worst case $\left(2^{n}\right.$ or $2^{n^{\prime}}$, respectively), the number of checks actually executed by the approach $(\# \sqrt{ })$, and the runtime for the detailed analysis (TIME) are given. Additionally the number of "non relevant" constraints $(|D C|)$ and self-contradictory constraints $(|Z|)$ obtained by the two properties are provided.

The results clearly show, that identifying all reasons without applying the properties leads to a large number of checks in the worst case (e.g. $2^{53} \geq 9.0 \cdot 10^{15}$ in example \#5). In contrast, when the properties are applied most of the constraints can be excluded for the analysis since they are "non relevant". This significantly reduces the number of checks to be performed at detailed analysis. Instead of all $2^{n}$ only $2^{n-|Z|-|D C|}$ checks are needed in the worst case (only 64 in example \#5). As a result the runtime of the detailed analysis is magnitudes faster when the properties are applied. Moreover, for the last three testcases the reasons can be determined within the timeout of 7200 CPU seconds only when the properties are applied.

\subsection{Real-life Example}

The constraint contradiction analysis algorithm has been evaluated using a real-life design example.

The Design Under Verification (DUV) is a PCIe root complex design with an AMD-proprietary host bus interface which is employed in a SoC recently developed by AMD. The root complex supports a number of PCIe links. The verification tasks are to show (1) that transactions are routed correctly from the host bus to one of the PCIe links and vice versa, (2) that the PCIe protocol is not violated and (3) that no deadlocks occur when multiple PCIe links communicate to the host bus at the same time.

Host bus and PCIe links are driven by Bus Functional Models (BFMs) which convert abstract bus transactions into
Table 1. Constraint characteristics

\begin{tabular}{||r|r|r|r|r|c||}
\hline$\#$ & BOOL & INT & LONG & BITS & CONSTR. $(n)$ \\
\hline \hline 1 & 10 & 8 & - & 328 & 15 \\
2 & 3 & 3 & 6 & 483 & 16 \\
3 & 10 & 10 & - & 330 & 26 \\
4 & 8 & 40 & - & 1,288 & 50 \\
5 & 5 & 30 & 15 & 1,925 & 53 \\
\hline
\end{tabular}

Table 2. Effect of using properties

\begin{tabular}{|c|c|c|c|c|c|c|c|c|c|c|c|}
\hline \multirow[b]{2}{*}{ \# } & \multirow[b]{2}{*}{$n$} & \multirow[b]{2}{*}{$|\mathcal{R}|$} & \multirow{2}{*}{$\begin{array}{l}\text { BDD } \\
\text { TIME }\end{array}$} & \multicolumn{3}{|c|}{ W/O PROPERTIES } & \multicolumn{5}{|c|}{ WITH PROPERTIES } \\
\hline & & & & $2^{n}$ & $\# \sqrt{ }$ & TIME & $|Z|$ & $|D C|$ & $2^{n^{\prime}}$ & $\# \sqrt{ }$ & TIME \\
\hline 1 & 15 & & 5.48 & 32,768 & 24,577 & 4.12 & 0 & 13 & 4 & 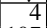 & 0.06 \\
\hline 2 & 16 & 3 & 14.90 & 65,536 & 26,883 & 11.25 & 1 & 8 & 128 & 107 & 0.04 \\
\hline 3 & 26 & 1 & 22.30 & $67,108,864$ & - & TO & 0 & 21 & 32 & 32 & 0.30 \\
\hline 4 & 50 & 3 & 35.96 & $>1.1 \cdot 10^{15}$ & - & TO & 0 & 42 & 256 & 190 & 2.10 \\
\hline 5 & 53 & 2 & 238.07 & $>9.0 \cdot 10^{15}$ & - & TO & 0 & 47 & 64 & 55 & 9.77 \\
\hline
\end{tabular}

the detailed signal wigglings on those buses. The abstract bus transactions are generated by means of random generators which are in turn controlled by constraints. Bus monitors observe the transactions sent into or from either interface and send them to checkers which perform the end-toend transaction checking of the DUV. The verification environment is implemented in SystemC 2.1, the SCV library, and SystemVerilog, with a special co-simulation interface synchronizing the SystemVerilog and SystemC simulation kernels. The constraint-random verification methodology was chosen in order to both reduce effort in stimulus pattern development and to get high coverage of stimulation corner cases. The PCIe and host bus protocol rules were captured in SCV constraint descriptions and are used to generate the contents of the abstract bus transactions driving the BFMs.

The PCIe constraint used to control stimulus generation within the PCIe transaction generator is a layered constraint. The lower level layer describes generic PCIe protocol rules and is comprised of a number of 16 constraint terms. They are shown in Figure 4(a) (denoted from $C_{0}$ to $\left.C_{15}\right)^{3}$. The meaning of the constraint variables is given in the table (Figure 4(b)). The upper level layer imposes userspecific constraints on the generic PCIe constraints (denoted by $C_{U_{i}}$ ) in order to generate specific stimulus scenarios. Generic PCIe constraints and user-defined constraints are usually developed by different verification engineers; the former by the designer of the test environment and the latter by the engineer who implements and runs the tests.

The engineer writing the tests and hence the user-specific constraints which are layered on top of the generic PCIe constraints is faced with the problem to resolve contradictions which are generated by imposing the user-defined constraints on the PCIe generic constraints. Given the complexity of the constraints, this is usually a non-trivial task. Two real-life examples of contradictions that are not easy to resolve by manual constraint inspection are depicted in Figure 4(c).

In the first example the user sets the maximum transaction length to a value greater than 128 bytes $\left(C_{U_{1}}\right)$, thereby causing a contradiction to constraint $C_{13}$, which states that the total transaction length must not exceed 128 bytes. In the second example, the user independently constrains the transaction address to byte address $4000\left(C_{U_{2}}\right)$ and the transaction length to 100 bytes $\left(C_{U_{3}}\right)$. While both values, viewed independently, are each perfectly legal (the address should be in 32 bit range and the transaction length is less than 128), an over-constraining occurs. The reason identified by our approach is $R_{1}=\left\{C_{12}, C_{U_{2}}, C_{U_{3}}\right\}$. By manual constraint inspection it is not immediately obvious that a PCIe protocol rule is violated when combining constraints $C_{U_{2}}$ and $C_{U_{3}}$. However, reason $R_{1}$ found for the contra-

${ }^{3}$ Bit operators are used as introduced in [6]. 
$C_{0} \Leftrightarrow($ addr_space ! = memory $\|(($ mem_addr_base $0<=$ addr $) \& \&(($ addr+length $)<=$ mem_addr_base $0+$ mem_size 0$)))$

$\|(($ mem_addr_base $1<=$ addr $) \& \&(($ addr+length $)<=$ mem_addr_base $1+$ mem_size 1$))) / /$ address boundaries for memory

$C_{1} \Leftrightarrow($ addr_space ! $=$ io $\|$ ((io_addr_base $<=$ addr $) \& \&((\operatorname{addr}+$ length $)<=$ io_addr_base + io_size $\left.\left.)\right)\right) / /$ address boundaries for io

$C_{2} \Leftrightarrow$ (addr_space != config $\|(($ cfg_base_addr $<=$ addr $) \& \&((\operatorname{addr}+$ length $)<=$ config_base_addr + config_size $\left.))\right) / /$ address boundaries for config

$C_{3} \Leftrightarrow \mathrm{be}[]<=0 x f / /$ valid byte enables are in $0 \times 0 . .0 x f$

$C_{4} \Leftrightarrow$ be[].len == length // generate as many byte enables as we have dword data

$C_{5} \Leftrightarrow$ data[].len $==$ length $/ /$ set data length

$C_{6} \Leftrightarrow \mathrm{cmd} !=$ read || posted $==$ false $/ /$ read transactions are always non - posted

$C_{7} \Leftrightarrow$ gen_host_trans.addr_space $==$ memory $\|$ (addr\&3)+length $<=4$

$/ /$ transactions to IO/config space are 1 dword (4bytes) only

$C_{8} \Leftrightarrow \operatorname{addr}<=0 x F F F F F F F F / /$ addresses are in 32 bit range

$C_{9} \Leftrightarrow$ addr_space==memory $\|$ addr_space==config $\|$ addr_space==io

$/ /$ only generate transactions in memory/IO/config space

$C_{10} \Leftrightarrow$ length $>0 / /$ requests must have length $>0$

$C_{11} \Leftrightarrow$ addr_space $==$ sr::mem $\|$ addr $<=0 x$ xFFFFFFF

// IO and config space are restricted to 32 bits

$C_{12} \Leftrightarrow($ addr\&4095) + length $<=4096 / /$ transactions must not cross $4 k$ page boundary

$C_{13} \Leftrightarrow$ (addr\&3) + length $<=128 / /$ keep transaction length to max. 128 bytes

$C_{14} \Leftrightarrow$ tkind $==$ request // generate requests only (not responses)

$C_{15} \Leftrightarrow \mathrm{msr}==$ false // do not generate MSR accesses

\begin{tabular}{|l|l|}
\hline addr & transaction address (64 bits) \\
\hline addr_space & $\begin{array}{l}\text { transaction address space } \\
\text { (memory,io,config) }\end{array}$ \\
\hline tkind & transaction kind (request,response) \\
\hline cmd & transaction command (read,write) \\
\hline msr & transaction is targeted at MSR space \\
\hline posted & transaction is posted (yes/no) \\
\hline length & transaction size in dwords \\
\hline be|] & $\begin{array}{l}\text { array of byte enables } \\
\text { (one per each dword data) }\end{array}$ \\
\hline data[] & array of dword (32 bit) data \\
\hline be[].len & length of byte enable array \\
\hline data[].len & length of data array \\
\hline $\begin{array}{c}\text { [io } \mid \text { mem } \mid \mathrm{cfg}]- \\
\text { addr_base0,1 }\end{array}$ & $\begin{array}{l}\text { io, memory and config space } \\
\text { window base addresses }\end{array}$ \\
\hline$[$ io $|\mathrm{mem}| \mathrm{cfg}]$ size0,1 & $\begin{array}{l}\text { io, memory and config } \\
\text { space window sizes }\end{array}$ \\
\hline
\end{tabular}

(a)

(b)

Example 1: $\quad C_{U_{1}} \Leftrightarrow$ length $>128$

Example 2: $\quad C_{U_{2}} \Leftrightarrow \operatorname{addr}==4000 \wedge C_{U_{3}} \Leftrightarrow$ length $==100$

(c)

Figure 4. PCle transaction generator constraint with examples

diction by our algorithm shows that when combining constraints $C_{U_{2}}$ and $C_{U_{3}}$, then PCIe protocol rule $C_{12}$ is violated: "A transaction must not cross a $4 \mathrm{k}$ page boundary". Our user constraints of transaction start address set to 4000 and transaction length of 100 bytes would result in addresses that cross a $4 \mathrm{k}$ page and therefore violate this constraint.

The algorithm described in this paper is able to identify exactly the violating constraint expressions for both examples in about 30 seconds. The PCIe constraint to be analyzed contained a total of 21 random variables to be solved which are constrained by 17 and 18 constraint expressions for the respective examples. The total bit count for the random variables amounted to 781 bits. Without such an analysis capability, we would have had to spend several hours on manual constraint inspection in order to identify the root cause for the constraint contradiction. Thus, a significant speed up of the contradiction debug cycle was achieved.

\section{Conclusions}

In this paper we have presented a fully automatic approach to analyze contradictory constraints that occur in constraint-based random simulation. After reformulating the overall constraint and building an abstraction, the selfcontradictory constraints and all "non relevant" constraints are determined in an initial step. Then for the small set of remaining constraints, all minimal reasons for a contradiction are computed efficiently and presented to the verification engineer. The minimality and completeness of the reasons allows to fully understand the over-constraining. Thus, the verification engineer is able to resolve the conflict in one single step. In total, as shown by industrial experiments, the debugging time is reduced significantly.

\section{References}

[1] R. R. Bakker, F. Dikker, F. Tempelman, and P. M. Wognum. Diagnosing and solving over-determined constraint satisfaction problems. In International Joint Conference on Artificial Intelligence, pages 276-281, 1993.

[2] J. Bergeron. Writing Testbenches Using SystemVerilog. Springer, 2006.
[3] R. Bryant. Graph-based algorithms for Boolean function manipulation. IEEE Trans. on Comp., 35(8):677-691, 1986.

[4] R. Dechter, K. Kask, E. Bin, and R. Emek. Generating random solutions for constraint satisfaction problems. In Eighteenth national conference on Artificial intelligence, pages 15-21, 2002.

[5] E. Goldberg and Y. Novikov. Verification of proofs of unsatisfiability for CNF formulas. In Design, Automation and Test in Europe, pages 10886-10891, 2003.

[6] D. Große, R. Ebendt, and R. Drechsler. Improvements for constraint solving in the SystemC verification library. In ACM Great Lakes Symposium on VLSI, pages 493-496, 2007.

[7] J. Huang. Mup: a minimal unsatisfiability prover. In ASP Design Automation Conf., pages 432-437, 2005.

[8] IEEE Std. 1666. IEEE Standard SystemC Language Reference Manual, 2005. [9] IEEE Std. 1800. IEEE SystemVerilog, 2005.

[10] C. N. Ip and S. Swan. A tutorial introduction on the new SystemC verification standard. White paper, 2003.

[11] M. A. Iyer. Race: A word-level ATPG-based constraints solver system for smart random simulation. Int'l Test Conf., pages 299-308, 2003.

[12] N. Kitchen and A. Kuehlmann. Stimulus generation for constrainted random simulation. In Int'l Conf. on CAD, pages 258-265, 2007.

[13] M. H. Liffiton and K. A. Sakallah. On finding all minimally unsatisfiable subformulas. In Theory and Applications of Satisfiability Testing, pages 173-186, 2005.

[14] M. N. Mneimneh, I. Lynce, Z. S. Andraus, J. P. Marques-Silva, and K. A. Sakallah. A branch and bound algorithm for extracting smallest minimal unsatisfiable formulas. In Theory and Applications of Satisfiability Testing, pages 467-474, 2005 .

[15] Y. Oh, M. Mneimneh, Z. Andraus, K. Sakallah, and I. Markov. Amuse: A minimally-unsatisfiable subformula extractor. In Design Automation Conf., pages 518-523, 2004.

[16] T. Petit, J.-C. Régin, and C. Bessière. Specific filtering algorithms for overconstrained problems. In International Conference on Principles and Practice of Constraint Programming, pages 451-463, 2001.

[17] J. Rose and S. Swan. SCV randomization version 1.0. 2003.
[18] F. Somenzi. CUDD: CU Decision Diagram Package Release 2.3.0. University of Colorado at Boulder, 1998.

[19] Synopsys Inc., CoWare Inc., and Frontier Design Inc., http://www.systemc.org. Functional Specification for SystemC 2.0.

[20] SystemC Verification Working Group, http://www.systemc.org. SystemC Verification Standard Specification Version 1.0e.

[21] J. Yuan, A. Aziz, C. Pixley, and K. Albin. Simplifying boolean constraint solving for random simulation-vector generation. IEEE Trans. on CAD of Integrated Circuits and Systems, 23(3):412-420, 2004.

[22] J. Yuan, C. Pixley, and A. Aziz. Constraint-based Verification. Springer, 2006.

[23] J. Yuan, K. Shultz, C. Pixley, H. Miller, and A. Aziz. Modeling design constraints and biasing in simulation using BDDs. In Int'l Conf. on CAD, pages 584-590, 1999

[24] L. Zhang and S. Malik. Validating SAT solvers using an independent resolutionbased checker: Practical implementations and other applications. In Design, Automation and Test in Europe, pages 10880-10885, 2003. 